\title{
Effects of Intraocular Lens Implantation without Viscoelastic Agents on Corneal Endothelial Cells
}

\author{
Hao Wang, Xueying Song, Huichun Yang, Qinghe Li, Chang'e Hu, Shaowen Qi \\ Department of Ophthalmology, PLA NO. 152 Hospital, Pingdingshan, China \\ Email: wh19871206@163.com
}

How to cite this paper: Wang, H., Song, X.Y., Yang, H.C., Li, Q.H., Hu, C.E. and Qi, S.W. (2018) Effects of Intraocular Lens Implantation without Viscoelastic Agents on Corneal Endothelial Cells. International Journal of Clinical Medicine, 9, 1-7. https://doi.org/10.4236/ijcm.2018.91001

Received: November 23, 2017

Accepted: January 6, 2018

Published: January 9, 2018

Copyright ( 92018 by authors and Scientific Research Publishing Inc. This work is licensed under the Creative Commons Attribution International License (CC BY 4.0).

http://creativecommons.org/licenses/by/4.0/

\begin{abstract}
Objective: To determine the protective effects of intraocular lens implantation without viscoelastic agents on corneal endothelial cells. Methods: Patients with age-related cataract were randomly divided into two groups: Group A (24 patients, 30 eyes) underwent phacoemulsification and intraocular lens implantation without viscoelastic agents, and group B (21 patients, 30 eyes) underwent phacoemulsification and intraocular lens implantation with viscoelastic agents. The corneal endothelial cell counts, percentages of hexagonal cells, and central corneal thicknesses were evaluated at 1 week, 1 month, and 6 months after surgery. Results: There was no significant difference in preoperative basic characteristics between the two groups $(p>0.05)$. The postoperative corneal endothelial cell count and percentage of hexagonal cells in both groups decreased compared with preoperative values at 1 week, 1 month, and 6 months after surgery, and the decrease of group A was significantly lower than that of group B at all time points $(p<0.05)$. The central cornea thickness in both groups increased at 1 week, 1 month, and 6 months after surgery, and there were no significant difference in the change of central cornea thickness between the two groups $(p>0.05)$. Conclusions: Compared with regular intraocular lens implantation, intraocular lens implantation without viscoelastic agents is less damaging to corneal endothelial cells, resulting in greater corneal safety.
\end{abstract}

\section{Keywords}

Intraocular Lens Implantation, Viscoelastic Agents, Corneal Endothelial Cells

\section{Introduction}

Cataract is the leading cause of blindness in China, and surgical removal is the 
main treatment [1]. Phacoemulsification was first applied in clinical treatments in 1967 [2] as a new technique in cataract surgery. After more than half a century of development, this surgical procedure has been significantly improved as a means to prevent blindness. Reducing damage to ocular tissues and providing a quick recovery of visual acuity have become the objectives of cataract surgery, which has been facilitated by the development of phacoemulsification. The change of number and morphology of corneal endothelial cells are evaluation indices used to assess ocular tissue damage [3] [4]. In this study, the effects of intraocular lens (IOL) implantation on corneal endothelial cells without viscoelastic agents during cataract phacoemulsification were compared with that of conventional IOL implantation with viscoelastic agents.

\section{Material and Methods}

\subsection{General Information}

The research described in this article followed the tenets of the Declaration of Helsinki and was approved by the ethics committee of the PLA NO.152 Hospital. Informed written consent was obtained from all patients. A total of 45 patients (60 eyes) diagnosed with age-related cataracts, from September 2014 to April 2015, who received phacoemulsification combined with IOL implantation in our hospital, and with lens nuclear grade of II-III, were recruited and treated with phacoemulsification with either IOL implantation without viscoelastic agents or by conventional intraocular lens implantation with viscoelastic agents. Group A without use of viscoelastic agents was comprised of 24 patients (30 eyes), including 11 males and 13 females, 54 - 78 years of age (average, 64.24 years). Group B that included conventional viscoelastic agents was comprised of 21 cases (30 eyes), including 10 males and 11 females, 52 - 84 years of age (average, 66.75 years). There was no significant difference in age and sex between the two groups $(p>0.05)$. Exclusion criteria included patients with high myopia, glaucoma, severe ocular fundus diseases (such as diabetic retinopathy and age-related macular degeneration), corneal macula or white plaque, a history of eye surgery or inflammation, intraoperative or postoperative complications (such as posterior capsular rupture), preoperative corneal endothelial cell counts $<1500$ cells $/ \mathrm{mm}^{2}$, or other serious systemic diseases [5].

\subsection{Equipment}

The corneal endothelial cell optical microscope (IOL Master; Carl Zeiss, Oberkochen, Germany), the WHITESTAR super emulsifying machine (AMO, Santa Ana, CA, USA), the disposable cornea keratome (Alcon, Fort Worth, TX, USA), a fluid flow management system, and an ultrasonic emulsification needle (AMO) were used.

\subsection{Surgical Procedures}

All surgeries were performed by the same surgeon with extensive experience in 
cataract phacoemulsification. Tropicamide eye drops were applied to achieve full mydriasis before surgery, followed by benoxinate eye drops for surface anesthesia. A speculum was used to open the eyelid, followed by observation of the lens nucleus hardness using a microscope, and a homemade chopping needle (a $1 \mathrm{ml}$ syringe needle bent backwards with a length of $2-2.5 \mathrm{~mm}$, obliquely facing down) was used to achieve an individualized length. A $2.8 \mathrm{~mm}$ keratome was used to make a clear superior corneal incision at the 2 o'clock position, $1 \mathrm{~mm}$ from the corneal limbus, followed by anterior chamber injection of viscoelastic agents. Capsulorhexis forceps were used to continuously perform circular capsulorhexis capsulotomy with a diameter of $5.5-6.0 \mathrm{~mm}$, followed by conventional water separation and stratification. The phacoemulsification energy was set at a conventional level of 50\%, with a negative pressure of ultrasonic emulsification at $350 \mathrm{mmHg}$, a bottle height of $80 \mathrm{~cm}$, and a perfusion flow of 30 $\mathrm{ml} /$ minute. A handmade chopping needle was used to split the lens into $6-8$ pieces by the cyclic sequential decomposition method. Chopping, nuclear separation, and emulsion of nuclear pieces were all performed in the capsule, followed by suction of the cortex and polishing of the capsule. In Group A, after the above steps, the lateral incision was perfused with balanced salt solution through the anterior vitrectomy infusion needle, and a nonspherical foldable IOL was inserted into the capsular bag, close to the incision. In group B, a viscoelastic agent was filled into the capsular bag and anterior chamber after polishing of the posterior capsule, followed by implantation of a nonspherical foldable IOL into the capsular bag, then suction of the viscoelastic agent and making the incision watertight to complete the surgery.

\subsection{Observation Index}

All patients underwent preoperative routine systemic examination and ophthalmological examination to exclude surgical contraindications that included a record of visual acuity, intraocular pressure, anterior chamber depth, axial length, corneal endothelial cell count, central corneal thickness, and lens nucleus hardness grade. During surgery, bottle height, negative pressure, flow rate, average composite ultrasonic energy (average ultrasonic energy and the effective phacoemulsification time), surgical approaches of intraoperative ultrasound emulsification, and the occurrence of complications were recorded. Postoperative corneal edema, visual acuity, intraocular pressure, corneal endothelial cell counts, and central corneal thicknesses were checked at 1 week, 1 month, and 6 months after surgery.

\subsection{Statistical Analysis}

SPSS Statistics software for Windows, version 19.0 (SPSS, Chicago, IL, USA) was used for statistical analyses. Corneal endothelial cell counts and central corneal thicknesses were expressed as $\bar{X} \pm s$, with an independent sample $t$-test used for comparison of preoperative data between the groups, and a paired $t$-test and an 
independent sample $t$-test used for postoperative intragroup and intergroup comparisons, respectively. Gender composition was analyzed using the $\chi^{2}$ test. A value of $p<0.05$ was considered statistically significant.

\section{Results}

\subsection{Comparison of Preoperative Data between the Two Groups}

The data of 60 eyes of 45 patients in the two groups are listed in Table 1 . There was no statistically significant difference in sex, age, corneal endothelium count, or central corneal thickness between the two groups $(p>0.05)$.

\subsection{Comparison of Corneal Endothelial Cell Count of the Two Groups before and after the Surgery}

There was no significant difference in the preoperative number of corneal endothelial cells between Group A and B $(p>0.05)$. The preoperative corneal endothelial cell count of group A was $2515 \pm 225$, and the number of corneal endothelial cells was significantly decreased at 1 week, 1 month, and 6 months after surgery $(p<0.05)$. The preoperative corneal endothelial cell count of group $\mathrm{B}$ was $2530 \pm 239$, and the number of corneal endothelial cells was significantly decreased at 1 week, 1 month, and 6 months after surgery $(p<0.05)$.

At 1 week, 1 month, and 6 months after surgery, the percentage of corneal endothelial cell loss of group A was significantly lower than that of group B ( $p<$ 0.05; Table 2).

\subsection{Comparison of Corneal Endothelial Cell Density of the Two Groups before and after Surgery}

There was no significant difference in preoperative corneal endothelial hexagonal cell density between the two groups $(p>0.05)$, which was significantly reduced in both groups at 1 week after surgery, and then increased at 1 month and 6 months after surgery, but was still significantly lower than the preoperative values. As shown in Table 3, at each postoperative time point, the corneal endothelial hexagonal cell density of group A was significantly greater than that of group B $(p<0.05)$.

Table 1. Comparison of preoperative data between the two groups of patients.

\begin{tabular}{cccccccc}
\hline \multirow{2}{*}{ Group } & $\begin{array}{c}\text { Number of } \\
\text { eyes }\end{array}$ & Sex & Age & Endothelial cell count & $\begin{array}{c}\text { Intraocular } \\
\text { pressure }\end{array}$ & $\begin{array}{c}\text { Anterior } \\
\text { chamber depth }\end{array}$ & $\begin{array}{c}\text { Ocular axial } \\
\text { length }\end{array}$ \\
\cline { 3 - 8 } & & $($ male/female $)$ & $(\bar{x} \pm s$, year $)$ & $\left(\bar{x} \pm s\right.$, cell/mm $\left.\mathrm{mm}^{2}\right)$ & $(\bar{x} \pm s, \mathrm{mmHg})$ & $(\bar{x} \pm s, \mathrm{~mm})$ & $(\bar{x} \pm s, \mathrm{~mm})$ \\
\hline $\begin{array}{c}\text { No viscoelastics } \\
\text { group }\end{array}$ & 30 & $11 / 13$ & $64.24 \pm 8.65$ & $2515.07 \pm 224.89$ & $15.54 \pm 1.48$ & $2.99 \pm 0.19$ & $23.09 \pm 0.27$ \\
$\begin{array}{c}\text { Conventional } \\
\text { viscoelastics group }\end{array}$ & 30 & $10 / 11$ & $66.75 \pm 9.38$ & $2530.30 \pm 238.72$ & $15.02 \pm 1.59$ & $3.04 \pm 0.16$ & $23.04 \pm 0.29$ \\
$t / \chi^{2}$ & $0.216^{*}$ & -0.387 & -0.418 & 0.755 & -0.730 & 0.422 \\
$p$ & 0.468 & 0.704 & 0.884 & 0.460 & 0.475 & 0.679 \\
\hline
\end{tabular}

Note: ${ }^{*}$ : is the value of $\chi^{2}$. 
Table 2. Corneal endothelial cell counts of the two groups before and after surgery (cell/ $\left.\mathrm{mm}^{2}, \bar{x} \pm s\right)$.

\begin{tabular}{|c|c|c|c|c|}
\hline & No viscoelastics group & Conventional viscoelastics group & $t$-value & $p$-value \\
\hline Before surgery & $2515.07 \pm 224.89$ & $2530.30 \pm 238.72$ & -0.418 & 0.884 \\
\hline 1 week after surgery & $2394.33 \pm 201.31$ & $2262.80 \pm 244.01$ & & \\
\hline Difference & $149.20 \pm 94.18$ & $267.50 \pm 63.18$ & -3.299 & 0.004 \\
\hline 1 month after surgery & $2310.90 \pm 221.95$ & $2161.80 \pm 232.19$ & & \\
\hline Difference & $204.10 \pm 106.79$ & $368.50 \pm 79.78$ & -3.900 & 0.001 \\
\hline 6 months after surgery & $2283.30 \pm 217.82$ & $2147.32 \pm 69.87$ & & \\
\hline Difference & $231.70 \pm 107.28$ & $393.63 \pm 61.27$ & -2.657 & 0.002 \\
\hline
\end{tabular}

Table 3. Percentage of hexagonal cells in the two groups before and after surgery $(\bar{x} \pm s)$.

\begin{tabular}{ccccc}
\hline & Before surgery & 1 week after surgery & 1 month after surgery & 6 months after surgery \\
\hline No viscoelastics group & $55.24 \pm 4.73$ & $50.27 \pm 5.87$ & $51.81 \pm 6.41$ & $52.67 \pm 6.28$ \\
Conventional viscoelastics group & $55.89 \pm 6.15$ & $43.67 \pm 7.84$ & $44.69 \pm 8.13$ & $44.93 \pm 8.12$ \\
t value & -0.262 & 2.132 & 2.174 & 2.385 \\
p-value & 0.796 & 0.015 & 0.021 & 0.032 \\
\hline
\end{tabular}

\subsection{Comparison of Central Corneal Thickness of the Two Groups before and after Surgery}

There was no significant difference in preoperative central corneal thickness between group A and group B $(p>0.05)$. At 1 week after surgery, the central corneal thicknesses of patients in both groups increased, but with no statistically significant difference compared with preoperative values $(p>0.05)$. At 1 month after surgery, the central corneal thicknesses vof patients in the two groups decreased, and at 6 months after surgery, the central corneal thickness of patients in both groups recovered to preoperative levels. As shown in Table 4, there was no significant difference in central corneal thicknesses $(p>0.05)$ between group A and group B at 1 week, 1 month, and 6 months after surgery.

\section{Discussion}

Along with the development of phacoemulsification and foldable IOLs, the safety and efficacy of cataract surgery have been greatly improved. Cataract surgery has now changed from sight restoration surgery to refractive surgery, and the main goal of current cataract surgery is quick visual recovery and the reduction of tissue injury.

Corneal endothelial cell density is an important parameter to assess ocular tissue injury. Corneal endothelial cells are monolayer cells attached to the posterior elastic layer of the cornea, which form the corneal-aqueous barrier to protect cornea tissue and to ensure corneal transparency [6]. Corneal endothelial cells cease mitosis during adulthood, so cell damage can only be repaired by expansion and migration of nearby corneal endothelial cells, and the density of 
Table 4. Central corneal thickness in the two groups before and after surgery $(\mathrm{mm}$, $\bar{x} \pm s)$.

\begin{tabular}{ccccc}
\hline & $\begin{array}{c}\text { No viscoelastics } \\
\text { group }\end{array}$ & $\begin{array}{c}\text { Conventional } \\
\text { viscoelastics }\end{array}$ & $t$-value & $p$-value \\
\hline Before surgery & $539.60 \pm 29.74$ & $541.10 \pm 40.31$ & -0.095 & 0.926 \\
1 week after surgery & $549.30 \pm 37.98$ & $551.90 \pm 43.37$ & -0.143 & 0.888 \\
Difference & $9.70 \pm 11.08$ & $10.80 \pm 6.53$ & -0.270 & 0.791 \\
1 month after surgery & $544.40 \pm 39.94$ & $546.50 \pm 42.57$ & -0.114 & 0.911 \\
Difference & $4.80 \pm 12.80$ & $5.40 \pm 10.12$ & -0.128 & 0.902 \\
6 months after surgery & $540.90 \pm 32.60$ & $543.10 \pm 41.77$ & -0.131 & 0.897 \\
Difference & $1.30 \pm 3.27$ & $2.10 \pm 2.83$ & -0.512 & 0.615 \\
\hline
\end{tabular}

corneal endothelial cells can therefore no longer be restored. Any form of cataract surgery will result in various degrees of damage to corneal endothelial cells, involving burn, ultrasonic vibration, fluid infusion, and mechanical injury of the lens nuclei [7].

Since the introduction of viscoelastic materials such as sodium hyaluronate in the 1970s, the filling, protection, and lubrication function of viscoelastic materials during phacoemulsification operations have been recognized and improved. However, our long-term clinical applications and observations suggest that use of viscoelastic agents still has the following problems: 1) When surgery is prolonged with increased steps, the possibility of losing more corneal endothelial cells increases and the corneal transparency decreases, 2) the possibility of early, medium-term, or tardive capsular block syndrome, 3) aspiration of viscoelastic materials from the rear surface of the IOL can sometimes cause posterior capsular rupture, 4) high postoperative intraocular pressure is mainly caused by mechanical obstruction of the trabecular meshwork by residual viscoelastic materials, and 5) early postoperative inflammatory reactions.

In the present study, we performed IOL implantation without viscoelastic agents, and compared the results with implantation using conventional viscoelastic agents. The losses of corneal endothelial cells at 1 week, 1 months, and 6 months after surgery were significantly reduced (all, $p<0.05$ ) in the absence of viscoelastic agents. The density of corneal endothelial hexagonal cells was significantly decreased compared with the preoperative values in both groups. But at all time points, the density of corneal endothelial hexagonal cells in the group without viscoelastic agents was significantly higher than that in the group with viscoelastic agents $(p<0.05)$. The thickness of the central cornea of both groups first increased, then decreased, and eventually returned to preoperative levels. Moreover, there was no significant difference in the central corneal thickness between the two groups at all time points before or after surgery $(p>0.05)$. These results suggest that IOL implantation without viscoelastic agents can cause less corneal endothelial damage, and therefore is safer. When surgery is performed without viscoelastic agents, in addition to a decreased impact on 
corneal endothelial cells, this surgical procedure also has the following advantages: 1) A reduced number of surgical steps and shortened surgical times, 2) no occurrence of the capsular block syndrome, 3) no posterior capsular rupture due to aspiration of viscoelastic agents from the rear surface of the IOL, 4) no high postoperative intraocular pressure due to residual viscoelastic materials, 5) reduced early postoperative inflammatory reactions, and 6) reduced surgical costs.

There are several important limitations in the current study. First, only endothielial cells were compared between the two groups, other measurements like visual acuity and intra ocular pressure were not determined. Second, the sample size our study was relatively small, future prospective studies with more participants would help to solve this problem.

\section{References}

[1] Li, F.M. and Li, J.Q. (1997) Applied Ophthalmology. People's Medical Publishing House, Beijing.

[2] Kelman, C.D. (1967) Phacoemulsification and Aspiration: A New Technique of Cataract Removal. A Preliminary Report. American Journal of Ophthalmology, 64, 23-25. https://doi.org/10.1016/0002-9394(67)93340-5

[3] Bourne, R., Minassian, D., Dart, J., Rosen, P., Kaushal, S. and Wingate, N. (2004) Effect of Cataract Surgery on Corneal Endothelium. Ophthalmology, 111, 679-685. https://doi.org/10.1016/j.ophtha.2003.07.015

[4] Hayashi, K., Yoshida, M. and Hayashi, H. (2009) Postoperative Corneal Shape Changes: Microincision versus Small-Incision Coaxial Cataract Surgery. Journal of Cataract \& Refractive Surgery, 35, 233-239. https://doi.org/10.1016/j.jcrs.2008.10.031

[5] Nayak, B.K. and Shkula, R.O. (2012) Effect Oncorneal Endothelialcell Loss during Phacoemulsification: Fortified Balanced Salt Solution versus Ringer Lactate. Journal of Cataract \& Refractive Surgery, 38, 1552-1558. https://doi.org/10.1016/j.jcrs.2012.04.036

[6] Mo, J.S., Zhang, C.W. and Zhang, T. (2012) Effects of Compound Sodium Chloride Infusion on Corneal Endothelium in Cataract Surgery. Chinese Journal of Ocular Trauma and Occupational Eye Disease, 34, 508-510.

[7] Belrdahlj, P., DeStafeno, J.J. and Kim, T. (2007) Cornea Wound Architecture and Integrity after Phacoemulsification: Evaluation of Coaxial, Microincision Coaxial, and Microincision Bimanual Techniques. Journal of Cataract \& Refractive Surgery, 33, 510-515. https://doi.org/10.1016/j.jcrs.2006.11.012 